University of Nebraska - Lincoln

DigitalCommons@University of Nebraska - Lincoln

Mechanical \& Materials Engineering Faculty

Publications

Mechanical \& Materials Engineering,

Department of

2008

\title{
Numerical Simulation of a Novel Blast Wave Mitigation Device
}

\author{
Zhenbi Su \\ University of Nebraska-Lincoln \\ Wen Peng \\ University of Nebraska-Lincoln \\ Zhaoyan Zhang \\ University of Nebraska-Lincoln, zzhang5@unl.edu \\ George Gogos \\ University of Nebraska-Lincoln, ggogos1@unl.edu \\ Reed Skaggs \\ Army Research Laboratory, Aberdeen Proving Ground, MD
}

See next page for additional authors

Follow this and additional works at: https://digitalcommons.unl.edu/mechengfacpub

Part of the Mechanical Engineering Commons

Su, Zhenbi; Peng, Wen; Zhang, Zhaoyan; Gogos, George; Skaggs, Reed; and Cheeseman, Bryan, "Numerical Simulation of a Novel Blast Wave Mitigation Device" (2008). Mechanical \& Materials Engineering Faculty Publications. 21.

https://digitalcommons.unl.edu/mechengfacpub/21

This Article is brought to you for free and open access by the Mechanical \& Materials Engineering, Department of at DigitalCommons@University of Nebraska - Lincoln. It has been accepted for inclusion in Mechanical \& Materials Engineering Faculty Publications by an authorized administrator of DigitalCommons@University of Nebraska Lincoln. 


\section{Authors}

Zhenbi Su, Wen Peng, Zhaoyan Zhang, George Gogos, Reed Skaggs, and Bryan Cheeseman 


\title{
Numerical simulation of a novel blast wave mitigation device
}

\author{
Zhenbi Su ${ }^{\mathrm{a}}$, Wen Peng ${ }^{\mathrm{a}}$, Zhaoyan Zhang ${ }^{\mathrm{a}, *}$, George Gogos ${ }^{\mathrm{a}}$, \\ Reed Skaggs ${ }^{b}$, Bryan Cheeseman ${ }^{\mathrm{b}}$

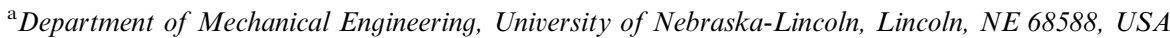 \\ ${ }^{\mathrm{b}}$ Army Research Laboratory, Aberdeen Proving Ground, MD 21005, USA
}

Received 10 August 2006; received in revised form 5 March 2007; accepted 4 April 2007

Available online 21 April 2007

\begin{abstract}
This paper proposes a novel blast wave mitigation device, consisting of a piston-cylinder assembly. A shock wave is induced inside the device when it is subject to a blast wave. The shock wave propagates inside the device and is reflected repeatedly. The physical processes within the blast wave mitigation device are simulated numerically. Numerical predictions are in excellent agreement with analytical solutions for special cases of the investigated problem that are available in the literature. The peak pressure on the base of the device caused by the blast wave is studied using a number of design parameters. The numerical simulation shows that, although the transmitted impulse remains practically unchanged, the peak pressure of the blast wave can be reduced by as much as $98 \%$, or even higher, depending on the design parameters chosen.
\end{abstract}

(C) 2007 Elsevier Ltd. All rights reserved.

Keywords: Blast wave; Mitigation; Numerical simulation

\section{Introduction}

It is well known that the blast wave produced by detonation of an explosive is characterized by extremely high peak pressure and short duration [1]. A blast wave can easily penetrate through hardened structures without much attenuation as a stress wave, which can be very damaging to military personnel and to structures. Extensive research has been conducted to reduce the damage potential of blast waves, which mainly depends on its peak pressure. Blast absorbing materials and heterogeneous systems are the two main approaches to mitigate the blast wave.

The list of blast absorbing materials typically includes granular, particulate matter, porous material, foam and water. Granular, particulate matter, porous material and foam were studied by Nesterenko [2]. Different materials used to mitigate blast waves were analyzed. The momentum and energy of a blast wave can be absorbed by these "soft" condensed materials. It was found that density, porosity and relative geometrical size of the so-called

\footnotetext{
*Corresponding author. Tel.: + 1402472 4993; fax: + 14024721465 .

E-mail address: zzhang5@unl.edu (Z. Zhang).
}

"soft" condensed matter are the main parameters determining the effectiveness of blast wave mitigation. Pfannes et al. [3] investigated a tapered chain of elastic beads under impulse loading. The elastic beads act as an absorber of kinetic energy or impulse and can reduce it by about $30 \%$. The energy absorption is affected by the restitution coefficient, the size of the particles and the tapering ratio. Edwards and Palmer [4] carried out experiments to determine the blast mitigation characteristics of particulate materials. They employed particulate materials with different densities, sizes and distributions as a mitigation layer. Results showed that the mitigation of an explosion is enabled largely by the consolidation of low-density particulate matter into compacts of greater density. Mitigation effects decrease with average particulate size for particulate material with low area densities.

Panczak et al. [5] studied the blast wave mitigation of aqueous foams. It was noted that many reflections off the foam/air interface produce a complicated waveform in the aqueous foam, which rapidly decays into the air region. Pressure drop occurred at the foam/air interface depths for small-sized foam. Numerical results were compared with experimental results and showed that the impedance 


\begin{tabular}{|c|c|c|}
\hline \multicolumn{2}{|c|}{ Nomenclature } & ratio of specific heats \\
\hline$a$ & speed of sound & coordinate attached on the moving piston \\
\hline$A$ & cross-sectional area of piston & density \\
\hline$e$ & total energy per unit volume & time in transformed coordinates \\
\hline$F$ & flux vector & \\
\hline$I$ & impulse per unit area & \multirow[t]{2}{*}{ Subscripts } \\
\hline$L$ & length of cylinder & \\
\hline$m$ & mass of piston & initial condition \\
\hline$M$ & Mach number & blast wave \\
\hline$P$ & pressure & base of device \\
\hline$\Delta P$ & initial shock wave strength & space step \\
\hline$R$ & gas constant & piston \\
\hline$t$ & time & \\
\hline$T$ & temperature & \multirow[t]{2}{*}{ Superscripts } \\
\hline$u$ & velocity & \\
\hline$U$ & variable vector & intermediate time step \\
\hline$x$ & cartesian coordinate & time step \\
\hline
\end{tabular}

mismatch between the air and foam is an important factor. $\mathrm{Li}$ and Meng [6] investigated the blast mitigation behavior of cellular materials by using a one-dimensional massspring model. It was indicated that the transmitted pressure could be attenuated by the foam layer if the input blast load is below a critical value. Full-scale field tests have been carried out by Hanssen et al. [7] to investigate the behavior of aluminum foam panels subjected to blast loading. Foam panels were attached to the bob of a ballistic pendulum. The energy and impulse transfer from the blast loading can be calculated through the maximum swing of the pendulum. An analytical solution was proposed to analyze the experiments and results showed that surface effects could affect the energy and impulse transfer.

Water has also been used to reduce the damage caused by blast waves. Gelfand and Silnikov [8] proposed an attenuation method that uses a liquid layer confined within an elastic envelop. Their results showed that the blast wave pressure attenuation coefficient depends on the distance from the blast epicenter to the point of measurement. Shin et al. [9] numerically simulated the mitigation effects of watershield on air blast wave. The magnitude of peak pressure and the shock wave arrival time were analyzed. The results showed that water medium delays the shock front and reduces the magnitude of initial peak shock pressure by $40 \%$. Chong et al. [10] numerically simulated the blast mitigation process by water using a threedimensional model. Results from their numerical simulations showed good agreement with experimental data for cases with and without water. The peak pressure can be reduced by more than $50 \%$ with water in comparison with the case without water.

Heterogeneous systems have also been designed to attenuate blast waves. Chapman et al. [11] examined the effect of geometrical parameters of a blast wall to protect a target structure. The authors used one-tenth scale to assess the sensitivity of blast parameters and measured the overpressure of the target behind the blast wall. The results showed the relationship between blast mitigation and geometrical configuration of the wall. Rose et al. $[12,13]$ measured the overpressure behind a one-tenth scale vertical blast wall. The height and distance of the wall affect the mitigation effectiveness. Results showed that the overpressures behind the wall are $30-60 \%$ of those without a wall.

It can be seen that conventional blast wave mitigation devices mainly use incompressible substances for blast wave protection. In this paper, a novel blast wave mitigation device is proposed to take advantage of the blast wave mitigation within a compressible substance. The device consists of a piston-cylinder assembly, which is shown in Fig. 1. Air or other gases with more desirable
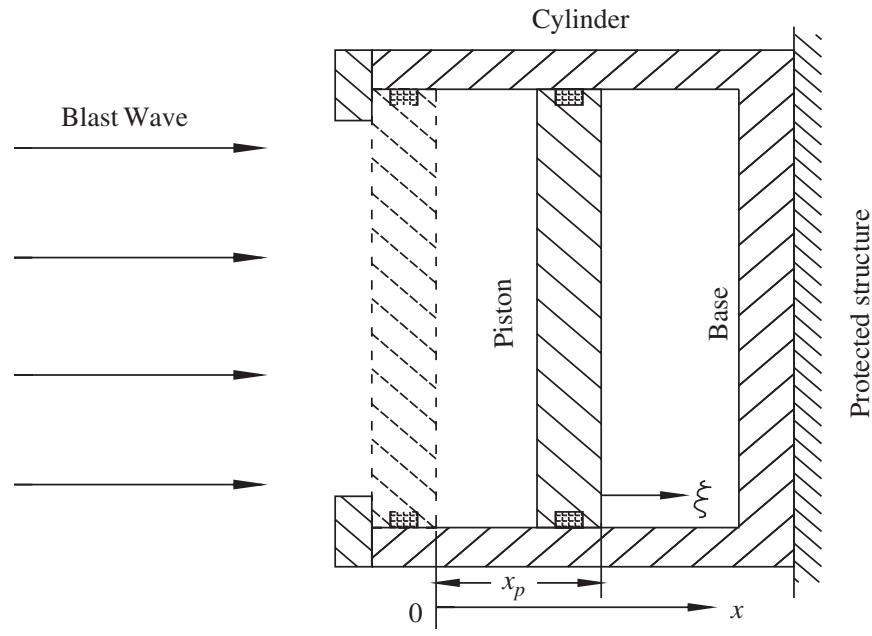

Fig. 1. Schematic of the blast wave mitigation device. 
characteristics may be used within the blast wave mitigation device. It should be noted that the piston-cylinder assembly has been used in dampers in many engineering systems. However, there are significant differences between physical mechanisms involved in the piston damper and the blast wave mitigation device. The piston dampers are used to diminish mechanical vibration. There is a blow-off valve to modulate the pressure inside the damper. The physical process involved is an equilibrium process over a long duration. Viscosity of the fluid inside the piston damper plays a significant role. In the blast wave mitigation device, because there is no blow-off valve, the viscosity of the fluid is not important for the blast wave mitigation device. Since the blast wave is of extremely high pressure and short duration, the compressible substance inside the blast wave mitigation device does not have sufficient amount of time to achieve equilibrium when the device is subject to a blast wave. A shock wave is induced by the blast wave impact. The shock wave propagates within the blast wave mitigation device and is reflected repeatedly. The repeated reflection of the shock wave within the blast mitigation device significantly increases the duration of the force on the base of the cylinder over that of the blast wave. Because the impulse of the blast wave is almost conserved, this results in a significant decrease of the force on the base of the cylinder. The essence of the device is to reduce a highpressure short-duration impact to a low-pressure longduration impact. Hence, a continuous grid of the proposed device can be used to cover the surface of military structures, to significantly reduce personnel casualties and equipment damage.

\section{Problem formulation and solution approach}

The mathematical model of the blast wave mitigation device is given in the following section. The shock wave propagation process inside the piston-cylinder assembly can be described by one-dimensional Euler equations in conservative form:

$\frac{\partial U}{\partial t}+\frac{\partial F}{\partial x}=0$

where $U$ is the variable vector and $F$ is the flux vector. These are given by

$U=\left(\begin{array}{c}\rho \\ \rho u \\ \rho e\end{array}\right), \quad F=\left(\begin{array}{c}\rho u \\ \rho u u+P \\ u(\rho e+P)\end{array}\right)$,

where $t$ is time, $x$ is the coordinate in the axial direction, $\rho$ is the density, $P$ is the pressure, $u$ is the velocity and $e$ is the total energy per unit volume of the gas within the device. It is assumed that (1) the shock wave propagation process is adiabatic, (2) viscous effects are negligible, and (3) the gas within the device obeys ideal gas law given by $P=\rho R T$, where $R$ is the gas constant and $T$ is the temperature.
Due to the piston motion during the blast wave mitigation process, an adaptive boundary-fitted coordinate system is used in the numerical simulation (see Fig. 1). The spatial and temporal variables in physical space are transformed to computational space by $\tau=t$ and $\xi=x-x_{\mathrm{p}}$. Subscript $\mathrm{p}$ indicates the piston. The Euler equations are transformed to

$\frac{\partial U}{\partial \tau}-\frac{\partial U}{\partial \xi} \frac{\mathrm{d} x_{\mathrm{p}}}{\mathrm{d} t}+\frac{\partial F}{\partial \xi}=0$.

The piston obeys Newton's second law of motion

$m \frac{\mathrm{d}^{2} x_{\mathrm{p}}}{\mathrm{d} t^{2}}=A\left(P_{\mathrm{b}}-P_{\xi=0}\right)$,

where $m$ is the mass of the piston, $A$ is the cross-sectional area of the piston and $P_{\mathrm{b}}$ is the pressure of the blast wave. Subscript $\mathrm{b}$ indicates the blast wave. Friction between the piston and cylinder is assumed to be negligible.

The initial conditions are:

$\left\{\begin{array}{rl}P & =P_{0} \\ u & =u_{0} \\ T & =T_{0}\end{array} \quad(\tau=0,0 \leqslant \xi \leqslant L)\right.$,

where $L$ is the length of the cylinder and subscript 0 indicates the initial values of the flow and thermodynamic properties of the gas within the device. The boundary conditions [14] at the base of the device are:

$\left.\frac{\partial P}{\partial \xi}\right|_{\xi=L-x_{\mathrm{p}}}=0$

$\left.\frac{\partial \rho}{\partial \xi}\right|_{\xi=L-x_{\mathrm{p}}}=0$,

$\left.u\right|_{\xi=L-x_{\mathrm{p}}}=0$.

The boundary conditions at the moving piston are

$\left.\frac{\partial P}{\partial \xi}\right|_{\xi=0}=0$,
$\left.\frac{\partial \rho}{\partial \xi}\right|_{\xi=0}=0$,
$\left.u\right|_{\xi=0}=\mathrm{d} x_{\mathrm{p}} / \mathrm{d} t$.

The shock wave propagation process inside the blast wave mitigation device can be simulated using the Van Leer flux vector splitting scheme $[15,16]$. The flux vector splitting scheme splits the flux vector $F$ into forward and backward flux components $F^{+}$and $F^{-}$. The forward and backward flux components have characteristic velocities going forward and backward, respectively. A standard upwind scheme can then be used to add numerical dissipation to provide numerical stability. The flux vector splitting is done in terms of the local one-dimensional Mach number $M=u / a$, where $a$ is the local speed of sound. For supersonic flow, i.e., $|M| \geqslant 1$, we have [17]

$F^{+}=F, \quad F^{-}=0$ for $M \geqslant 1$, 
$F^{+}=0, \quad F^{-}=F \quad$ for $M \leqslant-1$.

For subsonic flow $|M|<1$,

$F^{ \pm}=\left\{\begin{array}{c}f^{ \pm} \\ f^{ \pm}[(\gamma-1) u \pm 2 a] / \gamma \\ f^{ \pm}[(\gamma-1) u \pm 2 a]^{2} /\left[2\left(\gamma^{2}-1\right)\right]\end{array}\right\}$

where

$f^{ \pm}= \pm \rho a[(M \pm 1) / 2]^{2}$.

The spatial derivatives of the flux vectors are approximated with backward and forward differencing using the monotone upstream-centered scheme for conservation laws (MUSCL) approach [17]. First-order upwind scheme is applied to the term $(\partial U / \partial \xi)\left(\mathrm{d} x_{\mathrm{p}} / \mathrm{d} t\right)$ in Eq. (3). A two-step Runge-Kutta explicit scheme is used to solve the conservation equations, given by

$$
\begin{aligned}
& U^{*}=U^{n}-\frac{\Delta t}{2}\left(-u_{\mathrm{p}} \frac{U_{i}-U_{i-1}}{\Delta \xi}+\frac{\partial F_{i}}{\partial \xi}\right)^{n}, \\
& U^{n+1}=U^{n}-\Delta t\left(-u_{\mathrm{p}} \frac{U_{i}-U_{i-1}}{\Delta \xi}+\frac{\partial F_{i}}{\partial \xi}\right)^{*},
\end{aligned}
$$

where $u_{\mathrm{p}}=\mathrm{d} x_{\mathrm{p}} / \mathrm{d} t$, superscripts $n$ and $n+1$ stand for the vectors at time steps $n$ and $n+1$, respectively, and superscript $*$ implies an intermediate time step. The variable vector at the intermediate time step is first calculated. The flux vectors and source vector are updated to the intermediate time step. A two-step Runge-Kutta explicit scheme is also used to solve the Newton's second law of motion for the piston, given by

$u_{\mathrm{p}}^{*}=u_{\mathrm{p}}^{n}+\frac{\Delta t}{2} \frac{A}{m}\left(P_{\mathrm{b}}-P_{\xi=0}\right)^{n}$,

$u_{\mathrm{p}}^{n+1}=u_{\mathrm{p}}^{n}+\Delta t \frac{A}{m}\left(P_{\mathrm{b}}-P_{\xi=0}\right)^{*}$.

The displacement of the piston is obtained through

$x_{\mathrm{p}}^{n+1}=x_{\mathrm{p}}^{n}+u_{\mathrm{p}}^{*} \Delta t$.

\section{Numerical model validation}

Numerical results were compared with analytical results to validate the numerical model. Numerical validation is done for two special cases. First, numerical results of shock wave propagation and reflection are compared with analytical one-dimensional inviscid results [18]. Second, the numerical results are compared with the composite solution for shock wave initiation and propagation within a viscous heat conducting gas [19]. The input parameters used in the numerical validation are listed in Table 1 . To render the problem tractable analytically, the piston was
Table 1

Input parameters for numerical model validation

\begin{tabular}{lllllll}
\hline$u_{\mathrm{p}}(\mathrm{m} / \mathrm{s})$ & $P_{0}(\mathrm{kPa})$ & $T_{0}(\mathrm{~K})$ & $u_{0}(\mathrm{~m} / \mathrm{s})$ & $R(\mathrm{~J} /(\mathrm{kg} \mathrm{K}))$ & $\gamma$ & $L(\mathrm{~m})$ \\
\hline 38.17 & 100 & 300 & 0 & 287 & 1.4 & 0.2 \\
\hline
\end{tabular}

kept at a constant velocity. This problem can then be solved analytically. The analytical solution of propagation and reflection of a shock wave is documented in detail in standard compressible flow textbooks, such as [18]. The movement of the piston causes a shock wave. The mass motion behind the shock wave has a velocity of $u_{\mathrm{p}}$

$u_{\mathrm{p}}=\frac{a_{1}}{\gamma}\left(\frac{P_{2}}{P_{1}}-1\right)\left(\frac{2 \gamma /(\gamma+1)}{P_{2} / P_{1}+(\gamma-1) /(\gamma+1)}\right)^{1 / 2}$,

where $a_{1}$ is the speed of sound in front of the shock wave and $\gamma$ is the specific heat ratio. $P_{2}$ and $P_{1}$ are the pressures behind and in front of the shock wave, respectively. For a given pressure ratio $P_{2} / P_{1}$ and speed of sound $a_{1}$, the corresponding values of temperature and density can be calculated through the shock wave equations. The velocity of the shock wave $W$ can be expressed by

$W=a_{1} \sqrt{\frac{\gamma+1}{2 \gamma}\left(\frac{P_{2}}{P_{1}}-1\right)+1}$.

The shock wave hits the base of the device and gets reflected. The Mach number of the reflected shock wave $M_{\mathrm{R}}$ can be determined by

$\frac{M_{\mathrm{R}}}{M_{\mathrm{R}}^{2}-1}=\frac{M_{\mathrm{S}}}{M_{\mathrm{S}}^{2}-1} \sqrt{1+\frac{2(\gamma-1)}{(\gamma+1)^{2}}\left(M_{\mathrm{S}}^{2}-1\right)\left(\gamma+\frac{1}{M_{\mathrm{S}}^{2}}\right)}$,

where $M_{\mathrm{S}}$ is the Mach number of the incident shock wave. Additional details can be found in [18].

Shock wave initiation and propagation within a viscous heat conducting gas driven by a moving piston can be solved by using the Navier-Stokes equations and Burgers equation. In a paper by Moran and Shen [19], the linearized Navier-Stokes equations were found to be valid up to the period of the initiation of the shock wave. The Burgers equation was applied to solve the shock wave propagation. This solution is applicable for constant and small piston Mach number $(\sim 0.1)$, but cannot be applied to the reflected shock wave.

The numerical validation is shown in Fig. 2, which shows the pressure, density, temperature and velocity within the device at three different instants. It can be seen that the motion of the piston induces a weak shock wave, which propagates toward the base of the device at supersonic speed. When the shock wave reaches the base, it is reflected back. The reflected shock wave travels towards the piston. The piston continues to move toward the base of the device, while the reflected shock wave hits the piston and is reflected again. The reflected shock wave now travels 

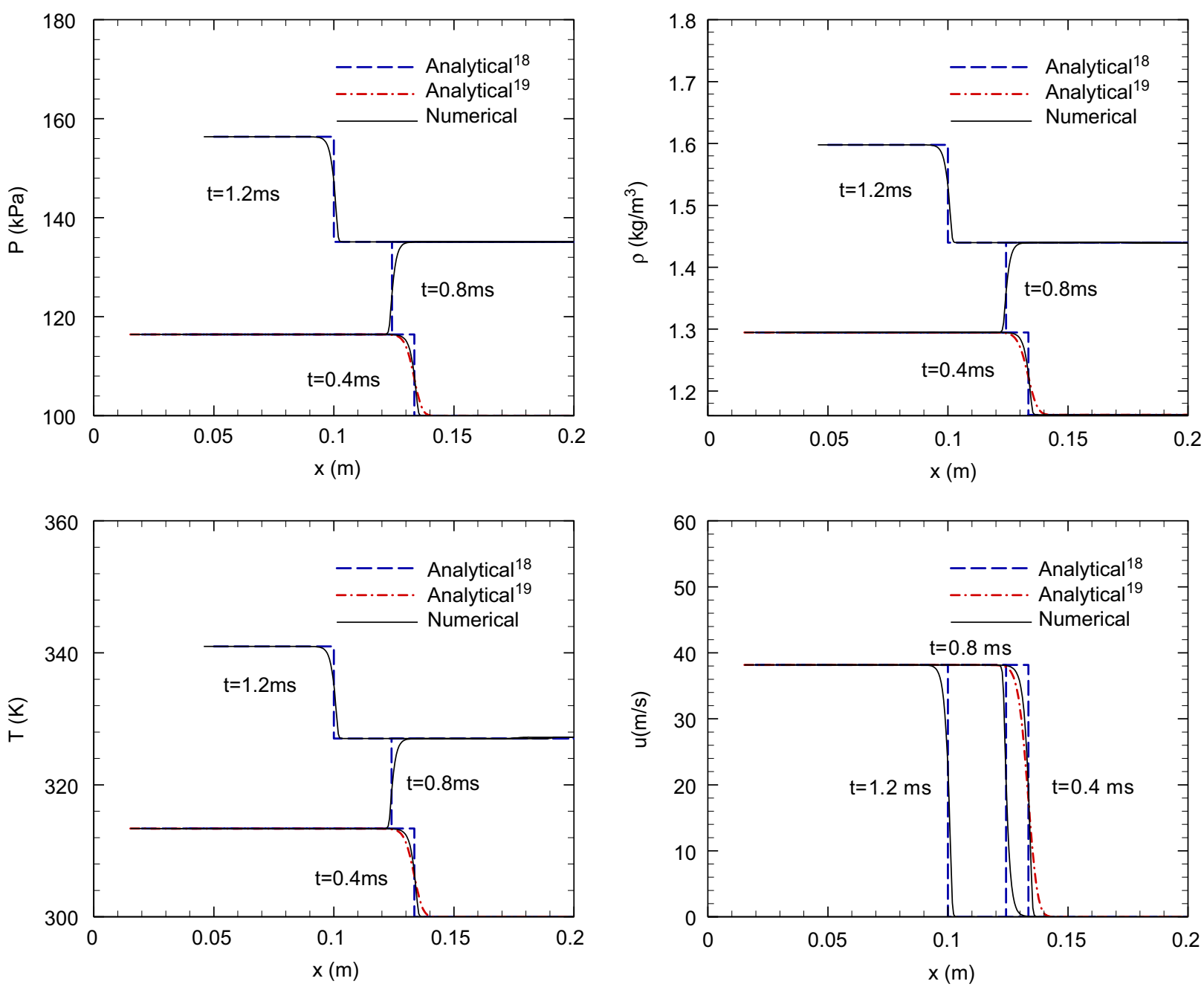

Fig. 2. Comparison of analytical solutions $[18,19]$ and numerical predictions for pressure, density, temperature and velocity profiles.

toward the base of the device again. Fig. 2 shows that the numerical results agree well with the analytical solutions. While the analytically obtained shock wave front shows very steep gradients in pressure, density, temperature and velocity, the shock wave is smeared in the numerical simulation. This is due to the numerical dissipation inherent in the flux vector splitting scheme.

\section{Results and discussion}

Numerical simulations were carried out to study the effects of the blast wave impulse, peak pressure and duration, as well as the length of the cylinder and the mass of the piston on the proposed blast wave mitigation device. The baseline design parameters of the blast wave mitigation device are listed in Table 2. Here $\delta$ is the thickness of the piston. The same values of flow and thermodynamic properties of air listed in Table 1 are used, except that the piston is initially stationary. A blast wave profile based on experimental data, as shown in Fig. 3, is used for the simulation. The experimental data were obtained at the Army Research Laboratory, Aberdeen Proving Ground,
Table 2

Baseline design parameters of the blast wave mitigation device

\begin{tabular}{llll}
\hline Piston (steel) & & & $\frac{\text { Cylinder }}{L(\mathrm{~m})}$ \\
\hline$\delta(\mathrm{m})$ & $A\left(\mathrm{~m}^{2}\right)$ & $m(\mathrm{~kg})$ & 0.2 \\
\hline 0.02 & 0.1398 & 21.98 & \\
\hline
\end{tabular}

MD. This blast wave corresponds to an explosion generated by $1.36 \mathrm{~kg}$ Pentolite at a distance of $0.26 \mathrm{~m}$, which has an impulse of $6000 \mathrm{~Pa}$ s. These parameters are kept constant in all simulations unless stated otherwise.

The numerical results of shock wave propagation inside the blast wave mitigation device are presented in Figs. 4 and 5. Fig. 4 shows the pressure, density, temperature and velocity of air within the blast wave mitigation device up to $0.2 \mathrm{~ms}$, which is also the duration of the blast wave. At the inception of the blast wave, the gage pressure outside of the blast wave mitigation device increases instantaneously to $132 \mathrm{MPa}$, while the pressure inside the device is $100 \mathrm{kPa}$. The pressure difference causes the piston to accelerate. As 


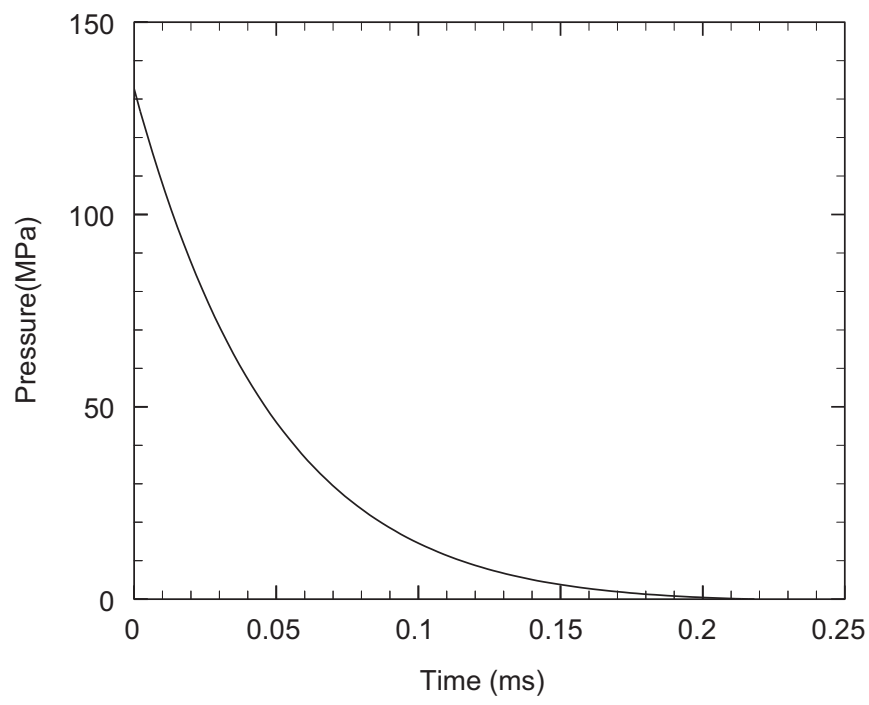

Fig. 3. Experimental data of the blast wave for numerical simulations.

air near the piston within the device is compressed, the pressure, density and temperature increase rapidly. Because of the short duration of the blast wave, the piston only achieves a maximum velocity of $38.17 \mathrm{~m} / \mathrm{s}$, which leads to a weak shock wave. At the end of the blast wave, the pressure outside the device decreases to $100 \mathrm{kPa}$. The piston however continues to move forward. The weak shock precedes the piston and propagates toward the base of the device with a supersonic speed. The shock wave strength is $16.41 \mathrm{kPa}$. The shock wave strength, usually defined as the ratio of the pressure behind and in front of the moving shock wave, is defined here as the pressure difference $\Delta P$ between pressures behind and in front of the shock wave. This definition shows the blast wave mitigation effects more conveniently. As the pressure within the blast wave mitigation device rises, the piston is slowed down. Fig. 5 shows the pressure, density, temperature and velocity of air within the blast wave mitigation device at four instants. The shock wave reaches the base and is reflected at approximately $0.59 \mathrm{~ms}$. The reflected shock wave is shown traveling in the opposite direction at $0.8 \mathrm{~ms}$. While the piston continues to move towards the base of the device, the reflected shock wave hits the piston and is reflected again at approximately $1.04 \mathrm{~ms}$. The shock wave now travels toward the base of the device, as shown at $1.2 \mathrm{~ms}$. Each time the shock wave is reflected, the pressure, temperature and density of air increase. The process repeats, until the piston comes to a complete stop. Because the piston moves towards the base of the device, the starting points for all curves in Fig. 5 also move toward the base of the device.

The displacement and velocity of the piston as a function of time are plotted in Fig. 6 as well. The piston accelerates during the blast wave and reaches the peak velocity of $38.17 \mathrm{~m} / \mathrm{s}$ at $t=0.2 \mathrm{~ms}$. The piston continues to move forward, beyond the duration of the blast wave. As the pressure inside the device increases, the piston decelerates. The piston comes to a complete stop at $t_{\mathrm{p}}=5.74 \mathrm{~ms}$ with a maximum displacement $x_{\mathrm{p}}$ of $0.193 \mathrm{~m}$.

The pressure on the base of the device reaches a maximum when the piston comes to a complete stop, after which, the pressure within the device causes the piston to move backward. A rarefaction wave is generated within the blast wave mitigation device and the pressure decreases as the piston moves backward. Since the peak pressure on the base of the device is the key parameter for evaluating mitigation effects of this blast wave mitigation device, the process of the piston moving backward and the propagation of the rarefaction wave is not presented. The blast wave pressure that impacts the piston and the pressure on the base of the device as a function of time are illustrated in Fig. 7. Both the blast pressure and pressure on the base of the device are gage pressures (gage pressure is also used in Figs. 8-11). The peak pressure on the base of the device is $9.882 \mathrm{MPa}$. It can be clearly seen that the device reduces the peak pressure of the blast wave by over $92 \%$. However, the duration of the pressure exerted on the base of the device increases by several orders of magnitude. The impulse of the blast wave is $6000 \mathrm{~Pa}$ s. The impulse on the base of the device is found to be $5979.6 \mathrm{~Pa}$ s. Thus, it is shown that the impulse of the blast wave is approximately conserved through the blast wave mitigation device. The slight difference is caused by the irreversibility of the shock wave propagation.

The response of the blast wave mitigation device to blast waves with an impulse of $6000 \mathrm{~Pa}$ s is presented next. The three cases are: (1) $P_{\mathrm{b}}=132 \mathrm{MPa}, t_{\mathrm{b}}=0.2 \mathrm{~ms}$, (2) $P_{\mathrm{b}}=$ $44 \mathrm{MPa}, t_{\mathrm{b}}=0.6 \mathrm{~ms}$, and (3) $P_{\mathrm{b}}=26.4 \mathrm{MPa}, t_{\mathrm{b}}=1 \mathrm{~ms}$. The pressure on the base of the device and displacement of the piston as a function of time are shown in Fig. 8. The curves for the three cases are nearly identical. The velocity of the piston depends on the impulse of the blast wave. Therefore, pistons obtain the same velocity history in all three cases. Since the velocity of the piston determines the initial shock wave strength, which relates to the increase of the pressure, temperature and density of air within the device, all these properties are dependent on the impulse of the blast wave. In conclusion, the response of the blast wave mitigation device is strongly dependent on the impulse of the blast wave. It is practically independent of the pressure and duration for a fixed impulse.

The effect of the blast wave pressure is presented in Fig. 9, which shows the pressure on the base of the device and displacement of the piston as a function of time under different peak blast wave pressures, while keeping a constant duration of the blast wave. Additional quantities are presented in Table 3 . Here $I_{\mathrm{b}}$ is the impulse of the blast wave. Larger pressure implies greater impulse. Fig. 9 shows that greater blast wave impulse induces larger piston velocity, which generates shock wave with greater shock wave strength. Consequently, the pressure within the device increases at a faster rate. Higher pressure inside the device causes the piston to decelerate faster. This leads to a 

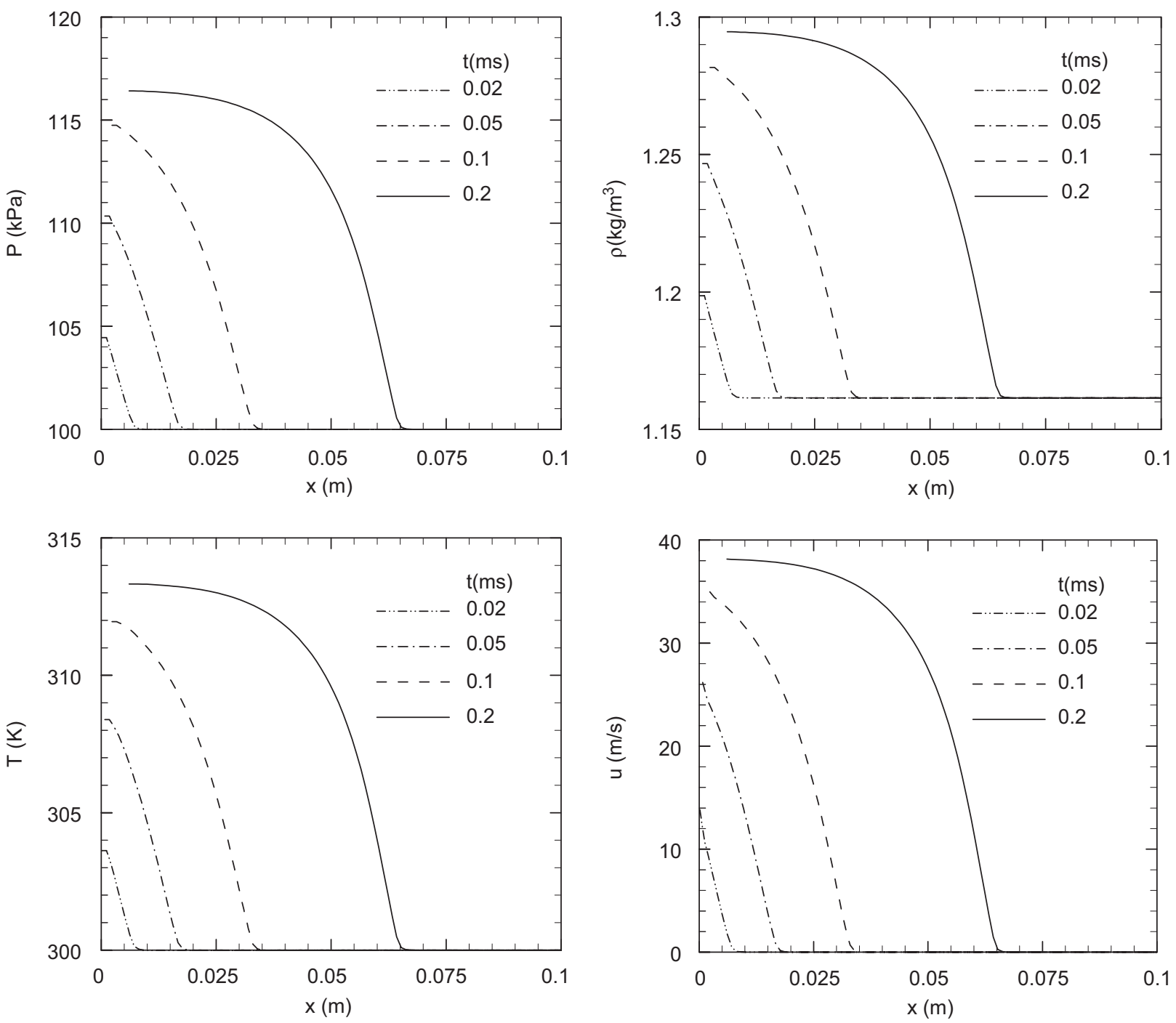

Fig. 4. Numerical predictions of pressure, density, temperature and velocity of air within the blast wave mitigation device up to $0.2 \mathrm{~ms}$.

shorter duration of piston movement. The numerically predicted impulse on the base of the device $I_{\mathrm{d}}$, displacements $x_{\mathrm{p}}$ and duration $t_{\mathrm{p}}$ of the piston movement, the initial shock wave strength induced by the piston $\Delta P$, the peak absolute pressure $P_{\max }$, peak temperature $T_{\max }$, and peak density $\rho_{\max }$ within the blast wave mitigation device are also listed in Table 3. It can be seen that as the blast wave pressure increases, the initial shock wave strength, and the maximum pressure, temperature and density within the blast wave mitigation device increase, while the duration of the piston movement decreases. Because of conservation of mass, the maximum displacement of the piston is related to the maximum density. As the maximum density increases, the displacement of the piston movement also increases. As expected, the impulse on the base of the device remains approximately equal to the impulse of the blast wave. As the blast wave pressure increases, the pressure on the base of the device is reduced by a smaller percentage through this blast wave mitigation device. It can be concluded that, as the blast wave pressure increases, the blast wave mitigation of the device becomes less effective. However, the reduction remains higher than $76.1 \%$ over this wide range of blast wave pressures.

The effect of the mass of the piston on the blast wave mitigation is also presented. Fig. 10 shows the pressure on the base of the device and displacement of the piston as a function of time for different piston thicknesses, which correspond to different mass of the piston. Additional quantities are presented in Table 4 . The momentum of the piston only depends on the impulse of the blast wave. Therefore lighter piston can obtain higher peak velocity under the same impulse. Higher peak velocity causes larger shock wave strength, which leads to increase of the pressure inside the device at a faster rate. As a result, higher pressure is obtained on the base of the device for lighter piston. This, together with the fact that the blast wave impulse is approximately conserved within the blast wave mitigation device, leads to a shorter duration of movement for lighter piston. It can also be seen that as the mass of the piston increases, the displacement of the piston 

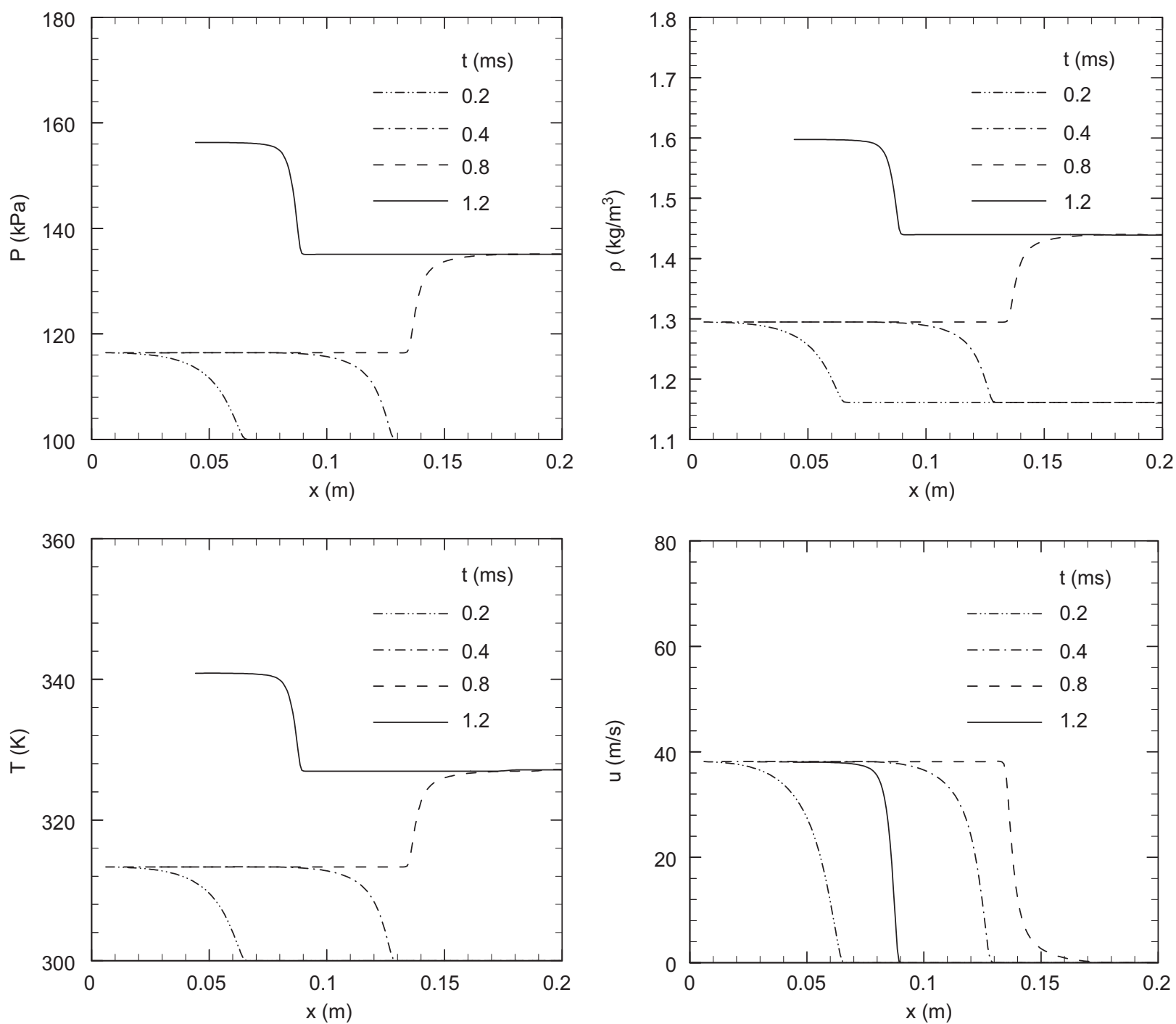

Fig. 5. Numerical predictions of pressure, density, temperature and velocity of air within the blast wave mitigation device up to $1.2 \mathrm{~ms}$.
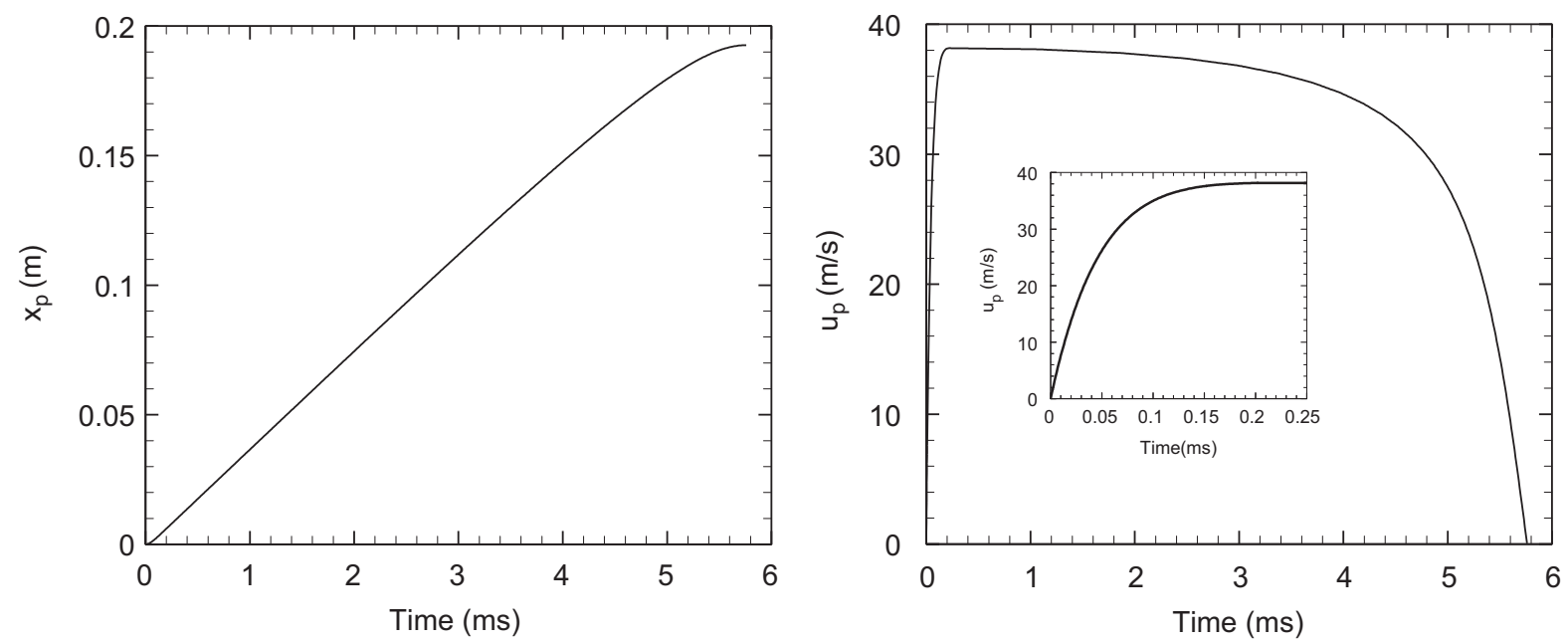

Fig. 6. The displacement and velocity of the piston as a function of time. 

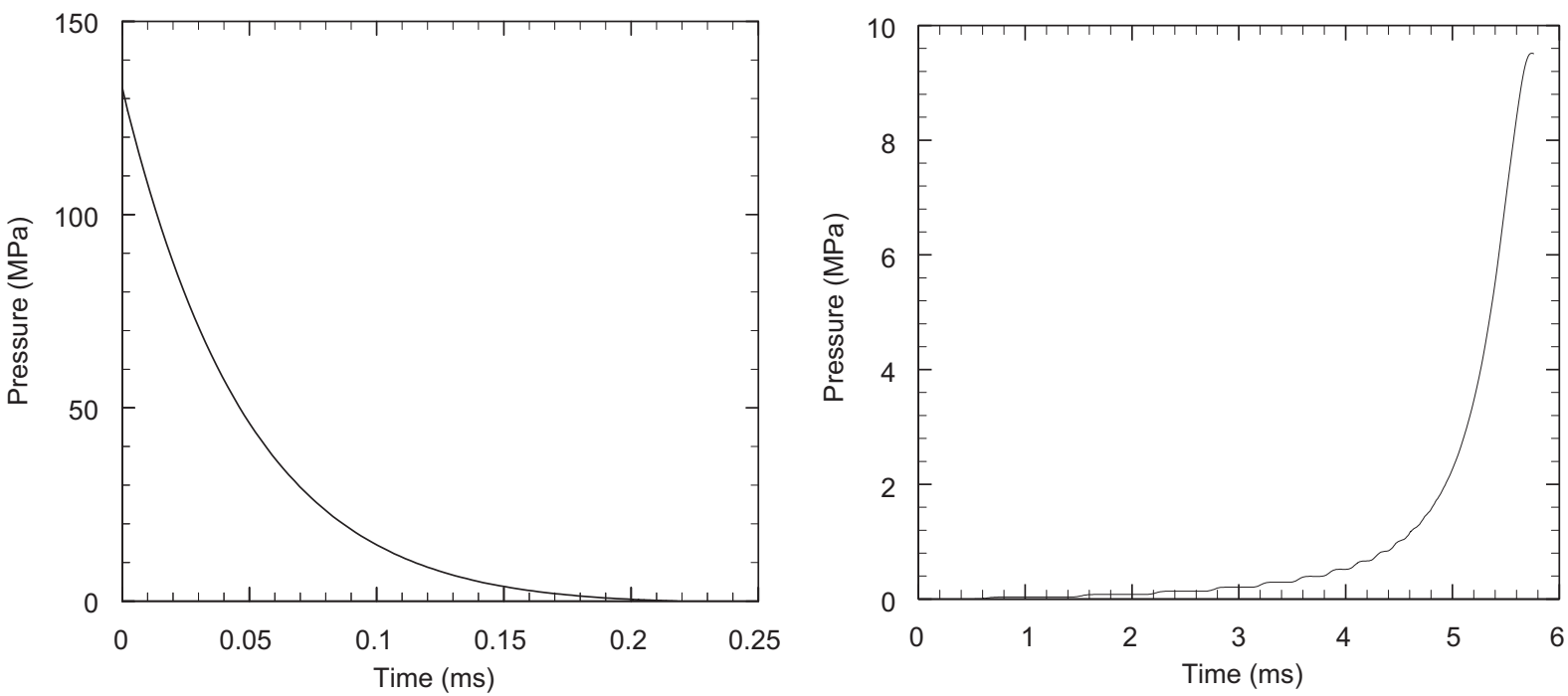

Fig. 7. Comparison of the blast wave pressure and the pressure on the base of the device.
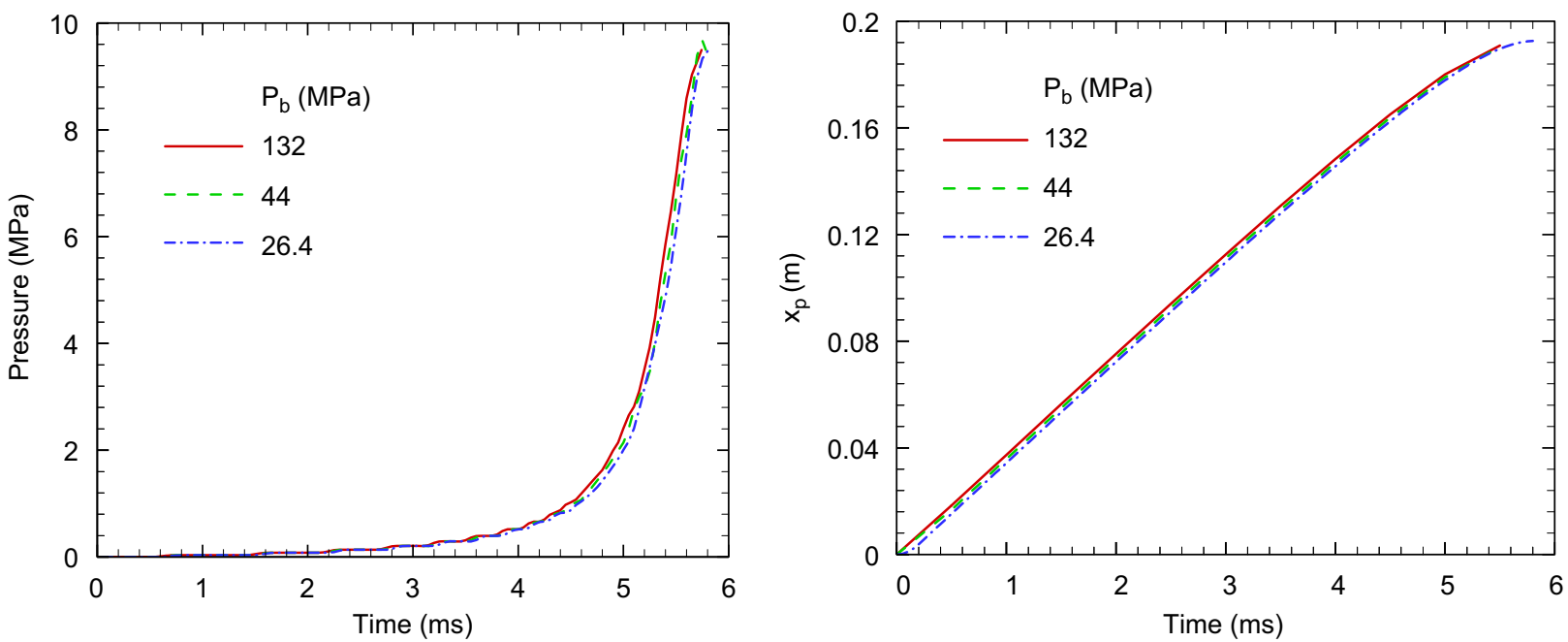

Fig. 8. The pressure on the base of the device and displacement of the piston for different peak pressures and durations keeping the blast wave impulse constant at $6000 \mathrm{Pas}$.
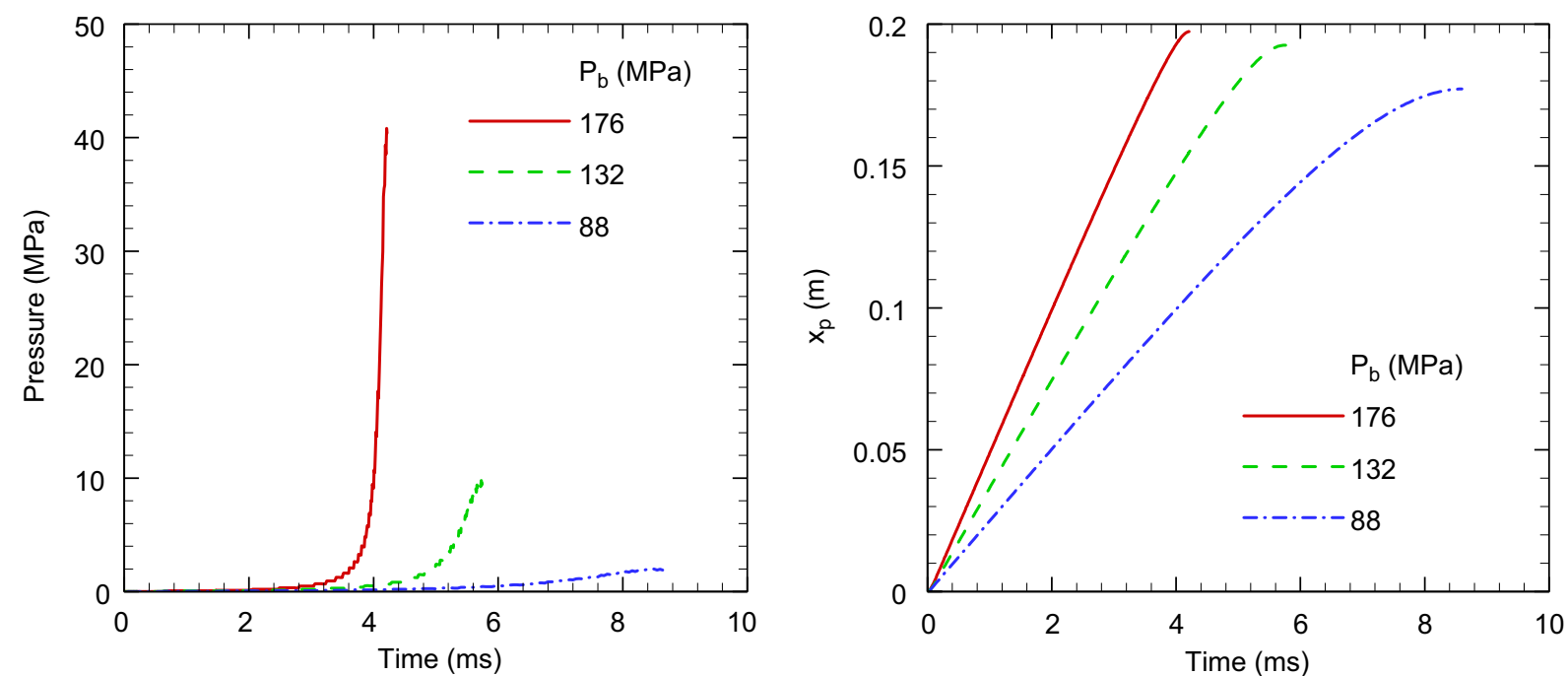

Fig. 9. The pressure on the base of the device and the displacement of the piston under different blast wave pressures $\left(t_{\mathrm{b}}=0.2 \mathrm{~ms}, L=0.2 \mathrm{~m}, \delta=0.02 \mathrm{~m}\right)$. 

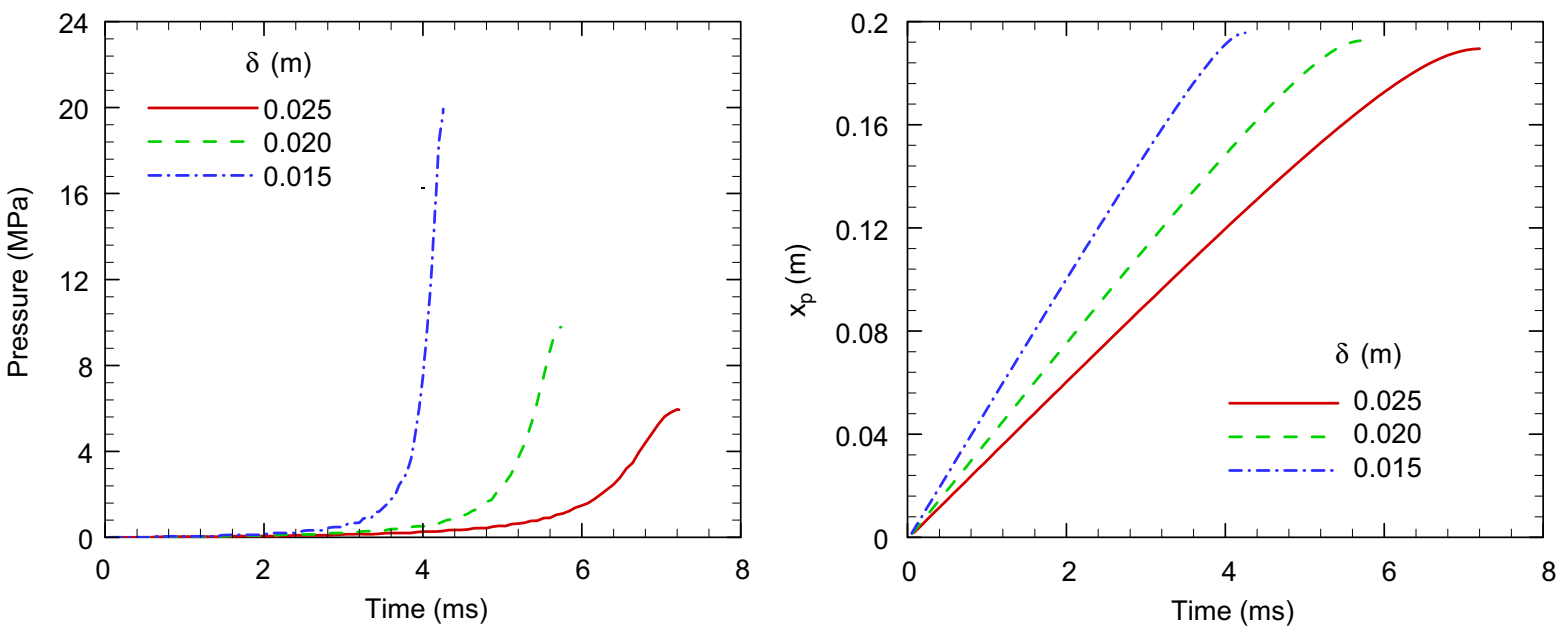

Fig. 10. The pressure on the base of the device and the displacement of the piston for different piston thicknesses $\left(P_{\mathrm{b}}=132 \mathrm{MPa}, t_{\mathrm{b}}=0.2 \mathrm{~ms}, L=0.2 \mathrm{~m}\right)$.
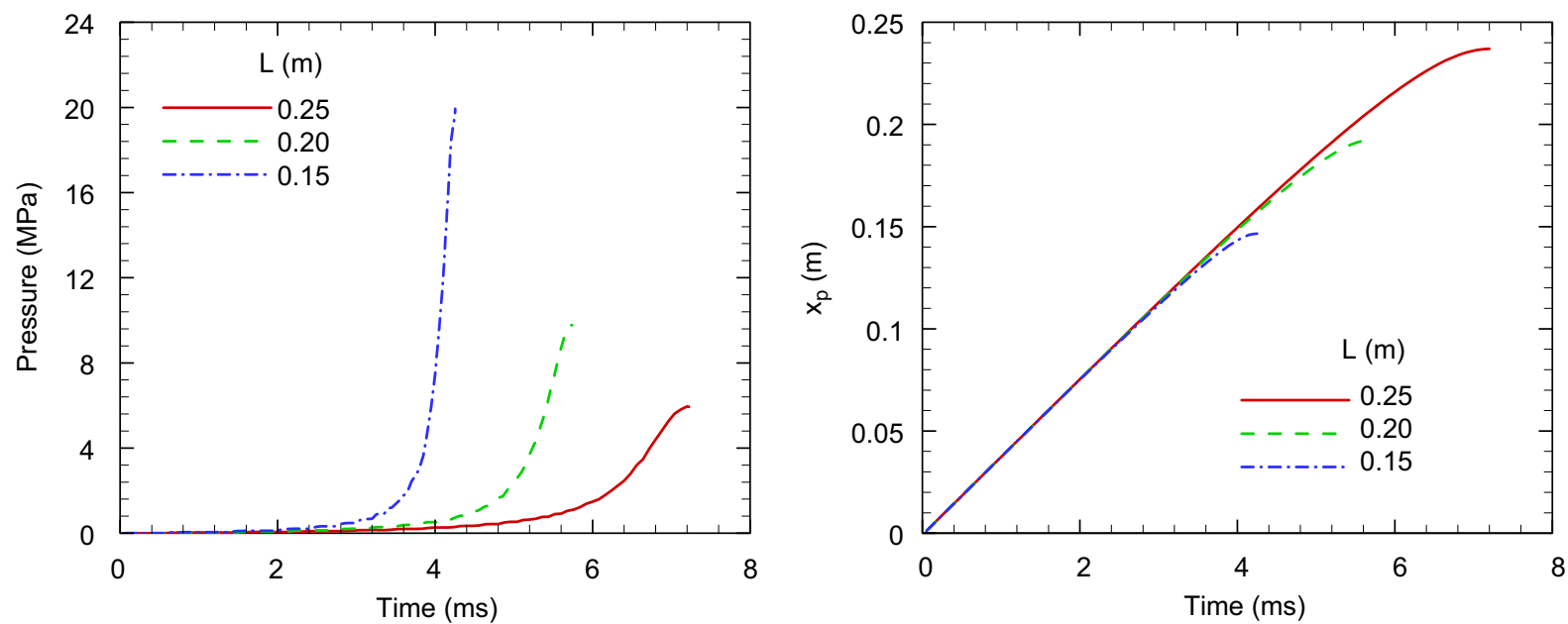

Fig. 11. The pressure on the base of the device and the displacement of the piston for different cylinder lengths $\left(P_{\mathrm{b}}=132 \mathrm{MPa}, t_{\mathrm{b}}=0.2 \mathrm{~ms}, \delta=0.02 \mathrm{~m}\right)$.

Table 3

Comparison under different blast wave pressures $P_{\mathrm{b}}$ with constant duration $t_{\mathrm{b}}\left(t_{\mathrm{b}}=0.2 \mathrm{~ms}, L=0.2 \mathrm{~m}, \delta=0.02 \mathrm{~m}\right)$

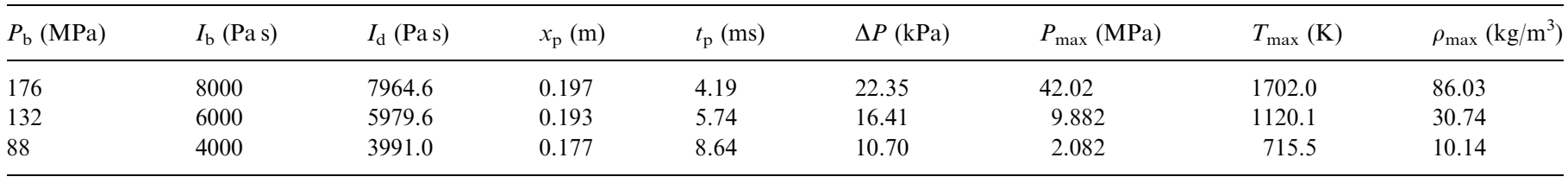

Table 4

Comparison for different thicknesses of the pistons $\delta\left(I_{\mathrm{b}}=6000 \mathrm{Pas}\right.$, $\left.P_{\mathrm{b}}=132 \mathrm{MPa}, t_{\mathrm{b}}=0.2 \mathrm{~ms}, L=0.2 \mathrm{~m}\right)$

\begin{tabular}{llllllrl}
\hline$\delta(\mathrm{m})$ & $I_{\mathrm{d}}(\mathrm{Pas})$ & $x_{\mathrm{p}}(\mathrm{m})$ & $t_{\mathrm{p}}(\mathrm{ms})$ & $\Delta P(\mathrm{kPa})$ & $P_{\max }(\mathrm{MPa})$ & $T_{\max }(\mathrm{K}) \begin{array}{l}\rho_{\max } \\
\left(\mathrm{kg} / \mathrm{m}^{3}\right)\end{array}$ \\
\hline 0.025 & 5985.9 & 0.189 & 7.22 & 12.96 & 6.036 & 971.1 & 21.66 \\
0.020 & 5979.6 & 0.193 & 5.74 & 16.41 & 9.882 & 1120.1 & 30.74 \\
0.015 & 5968.7 & 0.196 & 4.25 & 22.34 & 20.04 & 1376.6 & 50.72 \\
\hline
\end{tabular}

decreases. The maximum absolute pressure, temperature and density within the blast wave mitigation device also decrease with increasing piston mass. As expected, the impulse on the base of the device remains approximately equal to the impulse of the blast wave. As the mass of the piston increases, the pressure on the base of the device is reduced by a larger percentage. Therefore, the blast wave mitigation device becomes more effective, as the mass of the piston increases. 
Table 5

Comparison for different lengths of the cylinders $L\left(I_{\mathrm{b}}=6000 \mathrm{~Pa}\right.$, $\left.P_{\mathrm{b}}=132 \mathrm{MPa}, t_{\mathrm{b}}=0.2 \mathrm{~ms}, \delta=0.02 \mathrm{~m}\right)$

\begin{tabular}{llllllll}
\hline$L$ & $I_{\mathrm{d}}$ & $x_{\mathrm{p}}$ & $t_{\mathrm{p}}$ & $\Delta P$ & $P_{\max }$ & $T_{\max }$ \\
$(\mathrm{m})$ & $(\mathrm{Pa} \mathrm{s})$ & $(\mathrm{m})$ & $(\mathrm{kPa})$ & $\begin{array}{l}\rho_{\max }(\mathrm{kg} / \\
\left.\mathrm{m}^{3}\right)\end{array}$ \\
\hline 0.25 & 5977.0 & 0.237 & 7.22 & 16.41 & 6.094 & 975.3 & 21.77 \\
0.20 & 5979.6 & 0.193 & 5.74 & 16.41 & 9.882 & 1120.1 & 30.74 \\
0.15 & 5982.8 & 0.147 & 4.26 & 16.41 & 19.30 & 1356.6 & 49.57 \\
\hline
\end{tabular}

Finally, the effect of the cylinder length is presented in Fig. 11 and Table 5. Fig. 11 shows the pressure on the base of the device and the displacement of the piston as a function of time for different cylinder lengths. Table 5 shows that the initial shock wave strengths are the same, because the peak velocity of the piston is dependent on the mass of the piston and the blast wave impulse, and independent of the length of the cylinder. As the length of the cylinder increases, it takes longer time for the shock wave to propagate between the piston and the base of the device. This causes the pressure inside the device to increase at a slower rate. Therefore, the piston decelerates less rapidly and the duration of the piston movement increases. It can be seen that as the length of the cylinder increases, the displacement of the piston movement increases. While the displacement of the piston movement increases, air inside the device is actually compressed to a lesser degree with increasing cylinder length. Not surprisingly, the maximum density within the blast wave mitigation device decreases. The maximum absolute pressure and temperature within the blast wave mitigation device also decrease. Again, the impulse on the base of the device remains approximately equal to the impulse of the blast wave. As the length of the cylinder increases, the pressure on the base of the device is reduced by a larger percentage through this blast wave mitigation device. Thus, as the length of the cylinder increases, the blast wave mitigation device becomes more effective. In all cases presented in Tables $3-5$, the reduction of the blast wave pressure remains higher than $76.1 \%$.

\section{Conclusion}

A novel blast wave mitigation device is proposed to reduce the blast effects generated by explosives. This device consists of a piston-cylinder assembly. A shock wave is induced inside the device when it is subject to a blast wave. The shock wave propagates within the device and is reflected repeatedly. A numerical model of the blast wave mitigation device has been developed. The numerical model compares very well with analytical results. Numerical results show that the maximum pressure on the base of the device can be several orders of magnitude smaller than the pressure of the blast wave, while the duration of the force on the base of the device can be larger than the duration of the blast wave by orders of magnitude. The end result is that the impulse on the base of the device is approximately equal to the blast wave impulse. A parametric study of the device has been carried out. The effects of the blast wave impulse, pressure and duration were studied. It is also shown that the mass of the piston and the length of the cylinder are key parameters of this blast wave mitigation device. These parameters can be optimized to mitigate the impact of the blast wave.

\section{Acknowledgment}

Financial support for this study by Army Research Office Contract W911NF-04-2-0011 is gratefully acknowledged.

\section{References}

[1] Kinney G F, Graham K J. Explosive shocks in air. 2nd ed. New York: Springer; 1985.

[2] Nesterenko VF. Shock mitigation by 'soft' condensed matter. Mater Res Soc SymProc 2003;579:135-46.

[3] Pfannes J, Sen S, Chakravarti S, Surve FI. Energy absorption and recovery in tapered granular chains: small chains and low tapering. Mater Res Soc SymProc 2003;579:147-54.

[4] Edwards MR, Palmer ME. Mitigation of comminution effects of explosives by particulate materials. J App Phys 2003;93(5):2540-3.

[5] Panczak TD, Krier H, Butler PB. Shock propagation and blast attenuation through aqueous foams. J Hazard Mater 1987;14(3):321-36.

[6] Li QM, Meng H. Attenuation or enhancement-a one-dimensional analysis on shock transmission in the solid phase of a cellular material. Int J Impact Eng 2002;27(10):1049-65.

[7] Hanssen AG, Enstock L, Langseth M. Close-range blast loading of aluminium foam panels. Int J Impact Eng. 2002;27(6):593-618.

[8] Gelfand BE, Silnikov MV. The selection of the effective blast reduction method when detonating explosives. J Phys IV: JP 002; 12(7) $\operatorname{Pr} 7 / 371-4$.

[9] Shin YS, Lee M, Lam KY, Yeo KS. Modeling mitigation effects of watershield on shock waves. Shock Vib 1998;5(4):225-34.

[10] Chong WK, Lam KY, Yeo KS, Liu GR, Chong OY. Comparison of simulation's results with experiment on water mitigation of an explosion. Shock Vib 1999;6(2):73-80.

[11] Chapman TC, Rose TA, Smith PD. Reflected blast wave resultants behind cantilever walls: a new prediction technique. Int J Impact Eng 1995;16(3):397-403.

[12] Rose TA, Smith PD, Mays GC. The effectiveness of walls designed for the protection of structures against airblast from high explosives. Proc Inst Civil Eng Struct Build 1995;110(1):78-85.

[13] Rose TA, Smith PD, Mays GC. Protection of structures against airblast using barriers of limited robustness. Proc InstCivil Eng Struct Build 1998;128(2):167-76.

[14] Leveque RJ. Finite volume methods for hyperbolic problems. Cambridge: Cambridge University Press; 2002.

[15] Van Leer B. Flux-vector splitting for the Euler equations. Lect Notes Phys 1982;170:507-12.

[16] Anderson WK, Thomas JL, Van Leer B. Comparison of finite volume flux vector splittings for the Euler equations. AIAA J 1986;24(9): 1453-60.

[17] Mulder WA, Van Leer B. Implicit upwind methods for the Euler equations. AIAA paper no. 83-1930, 1983.

[18] Anderson JD. Modern compressible flow: with historical perspective. 3rd ed. New York: McGraw-Hill; 2003.

[19] Moran JP, Shen SF. On formation of weak plane shock waves by impulsive motion of a piston. J Fluid Mech 1966;25(4):705-18. 